\title{
Infant and young child feeding patterns in Kuwait: results of a cross-sectional survey
}

\author{
Manuel Carballo ${ }^{1,2, *}$, Noureen Khatoon ${ }^{1}$, Elizabeth Catherine Maclean ${ }^{2}$, \\ Nawal Al-Hamad ${ }^{3}$, Anwar Mohammad', Rehab Al-Wotayan ${ }^{4}$ and Smitha Abraham ${ }^{1}$ \\ 'Department of Public Health Research, Dasman Diabetes Institute, Kuwait City, Kuwait: ${ }^{2}$ International Centre for Migration \\ Health and Development, 11 Rue du Nant d'Avril, Geneva 1214, Switzerland: ${ }^{3}$ Food and Nutrition Department, \\ Ministry of Health, Kuwait City, Kuwait: ${ }^{4}$ Primary Health Care Department, Ministry of Health, Kuwait City, Kuwait
}

Submitted 2 December 2016: Final revision received 26 April 2017: Accepted 2 May 2017: First published online 5 July 2017

\begin{abstract}
Objective: The beneficial role of breast-feeding for maternal and child health is now well established. Its possible role in helping to prevent diabetes and obesity in children in later life means that more attention must be given to understanding how patterns of infant feeding are changing. The present study describes breastfeeding profiles and associated factors in Kuwait.

Design/Setting/Subjects: Interviews with 1484 recent mothers were undertaken at immunisation clinics across Kuwait. Descriptive analysis and binary logistic regression of results were performed.

Results: Rates of breast-feeding initiation in Kuwait were high (98.1\%) but by the time of discharge from hospital, only $36.5 \%$ of mothers were fully breast-feeding, $37 \cdot 0 \%$ were partially breast-feeding and $26.5 \%$ were already fully formulafeeding. Multiple social and health reasons were given for weaning the child, with $87.6 \%$ of mothers who had stopped breast-feeding completely doing so within 3 months postpartum. Nationality $(P<0.001)$, employment status 6 months prior to delivery $(P<0 \cdot 001)$, mode of delivery $(P=0 \cdot 01)$, sex of the child $(P=0.026)$ and breast-feeding information given by nurses $(P=0.026)$ were all found to be significantly associated with breast-feeding. Few women $(5.6 \%)$ got information on infant nutrition and feeding from nursing staff, but those who did were 2.54 times more likely to be still breast-feeding at discharge from hospital. Over $70 \%$ of mothers had enjoyed breast-feeding and $74 \%$ said they would be very likely to breast-feed again.

Conclusions: In Kuwait where the prevalence of both obesity and type 2 diabetes is growing rapidly, the public health role of breast-feeding must be recognised and acted upon more than it has in the past.
\end{abstract}

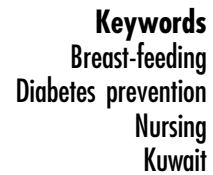

Since the WHO Collaborative Study on Breastfeeding ${ }^{(1,2)}$ and the WHO Collaborative Study on Quantity and Quality of Breast Milk in $1981^{(3)}$, the role of breast-feeding in enhancing the health and welfare of infants and mothers has been increasingly recognised by national as well as international bodies. Breast milk contains IgA antibodies, oligosaccharides and hormones that help protect against a range of allergies, acute respiratory infections, middle-ear infections, gastroenteritis, bacteraemia, meningitis, botulism and asthma ${ }^{(4)}$. There are also indications that it may help prevent childhood leukaemia, Crohn's disease and cardiovascular system disorders ${ }^{(4,5)}$.

Breast-feeding's global public health value has been highlighted recently in a Lancet series which estimated in low- and middle-income countries that $13 \%$ of deaths of children under 5 years of age and 820000 lives could be saved annually by breast-feeding via reducing infant respiratory infections by a third and infant diarrhoeal episodes by $50 \%{ }^{(6)}$. From a global perspective, 6 months of exclusive breast-feeding, as is recommended by $\mathrm{WHO}^{(7)}$, would both save hundreds of thousands of infant lives and significantly improve the health of millions of others $^{(8)}$. It has been further estimated that exclusive breast-feeding could translate into a saving of approximately \$US 300 billion per annum to the global economy ${ }^{(8)}$.

There is also evidence that breast-feeding may be protective against the development of obesity and type 2 diabetes mellitus among children ${ }^{(4,6,9-11)}$. A WHO meta-analysis has shown that breast-feeding confers a substantial reduction in the risk of diabetes (34\%) and 
overweight/obesity (24\%) later in life $e^{(12,13)}$. Conversely, other studies have suggested that the early use of infant formula may increase the risk of childhood type 1 diabetes mellitus ${ }^{(14)}$.

Breast-feeding also provides a number of benefits for the mother ${ }^{(10,15)}$. A study in the USA found that for each 'lifetime year' of breast-feeding, the risk of mothers developing diabetes was reduced by $15 \%$ (adjusted for diet, exercise and BMI) ${ }^{(16)}$. Lactation has also been shown to improve glucose metabolism in women who had gestational diabetes mellitus ${ }^{(16)}$ and delay subsequent development of type 2 diabetes mellitus by up to 10 years, especially in women who breast-fed for more than 3 months ${ }^{(17)}$.

Despite these now well-documented benefits and a global breast-feeding strategy that has been in place since $2002^{(7)}$, patterns of exclusive and partial breast-feeding continue to vary by time, place, demographic and socioeconomic factors ${ }^{(18)}$. Thus while there is evidence that rates of breast-feeding are improving in post-industrial countries such as the USA and the UK, especially among mothers with higher levels of education, rates of initiation of breastfeeding and the duration of exclusive and partial breastfeeding remain a concern in many parts of the world, especially so in low-income countries where the impact value of breast-feeding would be the highest ${ }^{(19)}$.

In Kuwait and the Gulf Cooperation Council (GCC) region, where patterns of obesity and diabetes in children, as well as adults, have changed dramatically in the past 30 years or so ${ }^{(20,21)}$, and where the age at first diagnosis of obesity and diabetes is decreasing, it is essential that as much as possible be known about factors possibly associated with this secular trend.

Because breast-feeding has been highlighted as helping to prevent obesity and diabetes, the present study set out to describe breast-feeding profiles and key factors in Kuwait.

\section{Methods}

\section{Study design}

Kuwaiti and non-Kuwaiti mothers with infants aged 12 months or less were recruited through a convenience sampling at twenty-five clinics where they came for routine infant immunisation between July and October 2015. Data were gathered using face-to-face interviews and a structured interview questionnaire designed and pre-tested by the Dasman Diabetes Institute. All interviews were conducted by medical students drawn from the University of Kuwait and trained in the use of the questionnaire. Interviews were conducted in Arabic or English according to the choice of the respondent. Approval for the project was granted by the Dasman Diabetes Institute Ethical Approval Board and the Kuwait Ministry of Health. Informed consent forms were gathered from all participants.

\section{Terminology}

The term 'fully breast-feeding' includes women who exclusively breast-fed (no formula or other liquids/solids) as well as those who breast-fed but also gave their infant water/juice (no formula or solid foods). The term 'partially breast-feeding' includes the use of breast milk and formula/solid foods as the main source of nutrition. The term 'fully formula-feeding' includes the use of formula milk as the primary source of food.

\section{Data analysis}

Of the total of 1484 mothers attending vaccination clinics with infants aged less than 12 months, twenty-seven did not meet the inclusion criteria of being over 21 years old; a further 130 were over the 0-12 month cut-off and were also excluded to avoid recall bias. Prior to the initiation of the study, power calculations were performed to ensure that a large enough sample was collected in order to perform meaningful statistical analyses. Analysis of data was done using the statistical software package IBM SPSS Statistics version 21, with descriptive statistics and $\chi^{2}$ tests for significance as initial forms of analysis. The significance level was defined as $P<0.05$ and $\mathrm{CI}$ are given to $95 \%$.

To assess the determinants of breast-feeding behaviour at the time of discharge from hospital, a multivariate binary logistic regression (forward conditional selection) analysis was performed. Independent variables were selected on the basis of univariate association or previous literature. Variables included in the analysis were: maternal age (age groups); employment prior to delivery (full-time, parttime, student; unemployed); educational level (primary, secondary, diploma/university); nationality (Kuwaiti, NonKuwaiti); number of children; weight gain during pregnancy (kilograms); mode of delivery (vaginally, elective caesarean section, emergency caesarean section); prematurity (born at term, born before 37 weeks); sex of child; skin-to-skin contact (immediately after delivery, or with no systematic skin-to-skin contact); information provided by paediatrician (yes, no); information provided by nurse (yes, no); information provided by friends (yes, no); information provided by mother/grandparents (yes, no); and information found myself (yes, no). Based on previous studies $^{(22)}$ indicating the minimum number of cases required for logistic regression results, the sample was large enough to ensure that relationships between independent variables and breast-feeding at discharge were identified and precise.

\section{Results}

\section{Demograpbic profile}

The age profile of infants was: $27 \%$ aged less than 3 months; $41 \%$ aged $3-5$ months; $21 \%$ aged 6-8 months; and $10 \%$ aged 9 months to 1 year. 
Almost half ( $49 \%$ ) of the mothers were Kuwaiti nationals and $51 \%$ were non-Kuwaitis. Given that expatriates constitute approximately $70 \%$ of the general population in Kuwait, non-Kuwaitis were under-represented. All six health administrative districts were covered by the survey but the proportions of respondents varied: $41 \%$ of the mothers were from the Hawalli district; others were from Jahra (27\%), Assima (13\%), Ahmadi (11\%), Farwaniya (6\%) and Mubarak Al-Kabeer (0.5\%). The majority of mothers were in the 26-30 years age group (40\%); $24 \%$ were $21-25$ years old; $24 \%$ were $31-35$ years old; and $12 \%$ were aged $\geq 36$ years. Approximately one-third (30\%) of the mothers had completed secondary education; over two-thirds (68\%) had gone on to diploma- or universitylevel education. Half $(50 \%)$ of the participants said they had not been employed or studying 6 months prior to giving birth; $36 \%$ had been in full-time employment; $13 \%$ had been in part-time employment or were students.

\section{Maternal bealth profile}

Self-reported maternal health varied irrespective of nationality or ethnic background. The self-reported mean BMI was 27.27 (CI 26.99, 27.55) kg/m² and the mean weight gain during pregnancy was 11.44 (CI 11.07, 11.81) $\mathrm{kg}$. Almost a third of all the deliveries were by caesarean section: $9.8 \%$ elective and $23.2 \%$ emergency. A further $61.8 \%$ gave birth normally without forceps or suction, and $5 \cdot 1 \%$ required forceps or suction.

\section{Breast-feeding pattern at discharge}

Rates of breast-feeding initiation were high (98.1\%) and in accordance with previous studies in Kuwait and the GCC region $^{(23,24)}$. By the time of discharge from hospital, however, only $36.5 \%$ of mothers said they were fully breast-feeding; another $37 \cdot 0 \%$ were partially breastfeeding; and $26.5 \%$ were already fully formula-feeding.

\section{Determinants of breast-feeding at discharge}

Associations with breast-feeding at time of discharge from hospital are summarised in Tables 1 and 2. The multivariate analysis highlighted the role of nationality $(P<0 \cdot 001)$, employment status 6 months prior to delivery $(P<0.001)$, mode of delivery $(P=0.01)$, sex of the child $(P=0.026)$ and breast-feeding information given by nurses $(P=0 \cdot 026$; see Table 2).

Culture is known to be an important determinant of breast-feeding ${ }^{(25)}$ and in the present study non-Kuwaiti mothers were $2 \cdot 19$ times more likely than Kuwaiti mothers to be breast-feeding at the time of discharge. Mothers who had been in part-time employment prior to delivery were less likely to be breast-feeding at discharge from hospital than mothers who had not been employed, while mothers who had vaginal deliveries were 1.54 times more likely to be breast-feeding than those who had an emergency caesarean section. The sex of the child also emerged as a possible determinant of breast-feeding practices; women with male babies were significantly more likely to be still breast-feeding at time of discharge (OR =1.39).

Where mothers got information and advice on infant feeding was also critical for mothers' decision making; over half $(59.5 \%)$ of the mothers mentioned their paediatrician as a key source of information. Other frequently stated sources were parents/grandparents (39.6\%), mothers' previous knowledge (40.7\%) and friends (9.6\%). Noticeably few women $(5.6 \%)$ referred to getting information from nursing staff, but those who did receive information from nurses were 2.54 times more likely to be still breast-feeding at time of discharge than mothers who did not name nurses as a key source of information.

Although it was not found to be significant in the multivariate regression model, younger mothers were more likely to be exclusively breast-feeding, while older mothers were more likely to be fully formula-feeding or at least partially breast-feeding at the time of discharge (Table 1 and Fig. 1).

Early skin-to-skin contact is known to enhance bonding and facilitate early suckling and continued breastfeeding ${ }^{(26)}$. Over half $(55.7 \%)$ of the mothers said they

Table 1 Participant characteristics according to breast-feeding pattern at discharge from hospital among a convenience sample of mothers $(n$ 1484) with infants aged 12 months or less, Kuwait, July-October 2015

\begin{tabular}{|c|c|c|c|}
\hline \multirow[b]{2}{*}{ Variable } & \multicolumn{3}{|c|}{$\begin{array}{l}\text { Breast-feeding pattern } \\
\text { at discharge from hospital }\end{array}$} \\
\hline & $\begin{array}{c}\text { Fully } \\
\text { breast- } \\
\text { feeding } \\
\text { (valid \%) }\end{array}$ & $\begin{array}{l}\text { Partially } \\
\text { breast- } \\
\text { feeding } \\
\text { (valid \%) }\end{array}$ & $\begin{array}{c}\text { Fully } \\
\text { formula- } \\
\text { feeding } \\
\text { (valid \%) }\end{array}$ \\
\hline Total & $36 \cdot 5$ & $37 \cdot 0$ & $26 \cdot 5$ \\
\hline \multicolumn{4}{|l|}{ Mother's age } \\
\hline $21-25$ years & 43.5 & 31.9 & 24.5 \\
\hline $26-30$ years & $36 \cdot 9$ & $36 \cdot 8$ & $26 \cdot 3$ \\
\hline $31-35$ years & $32 \cdot 9$ & 39.6 & $27 \cdot 5$ \\
\hline$\geq 36$ years & $27 \cdot 4$ & $42 \cdot 7$ & 29.9 \\
\hline \multicolumn{4}{|l|}{ Nationality } \\
\hline Kuwaiti & $24 \cdot 3$ & $43 \cdot 3$ & $32 \cdot 4$ \\
\hline Non-Kuwaiti & 48.3 & 31.0 & $20 \cdot 7$ \\
\hline \multicolumn{4}{|c|}{ Employment status 6 months prior to delivery } \\
\hline No employment or studies & 41.9 & 33.8 & $24 \cdot 3$ \\
\hline Full-time employed & 33.0 & $41 \cdot 8$ & $25 \cdot 3$ \\
\hline Part-time employed & $26 \cdot 1$ & 23.9 & $50 \cdot 0$ \\
\hline Student & $25 \cdot 0$ & 47.6 & $27 \cdot 4$ \\
\hline \multicolumn{4}{|l|}{ Education } \\
\hline Primary & $44 \cdot 0$ & $32 \cdot 0$ & $24 \cdot 0$ \\
\hline Secondary & $35 \cdot 0$ & $38 \cdot 8$ & $26 \cdot 3$ \\
\hline Diploma/university & $36 \cdot 1$ & $36 \cdot 9$ & $27 \cdot 0$ \\
\hline \multicolumn{4}{|l|}{ Skin-to-skin contact } \\
\hline $\begin{array}{l}\text { Without skin-to-skin } \\
\text { contact soon after delivery }\end{array}$ & $31 \cdot 0$ & $40 \cdot 8$ & $28 \cdot 2$ \\
\hline $\begin{array}{l}\text { With skin-to-skin contact } \\
\text { soon after delivery }\end{array}$ & $40 \cdot 7$ & $34 \cdot 0$ & $25 \cdot 3$ \\
\hline \multicolumn{4}{|c|}{ Sources of breast-feeding information } \\
\hline Nurses & $62 \cdot 3$ & $26 \cdot 1$ & $11 \cdot 6$ \\
\hline Paediatrician & 39.6 & 31.4 & 29.0 \\
\hline Friends & 30.5 & 41.5 & 28.0 \\
\hline Mother or grandparents & $35 \cdot 2$ & 38.0 & $26 \cdot 8$ \\
\hline Self-found & $36 \cdot 2$ & $36 \cdot 6$ & $27 \cdot 2$ \\
\hline
\end{tabular}


Table 2 Final forward conditional logistic regression model on determinants of breast-feeding at discharge from hospital among a convenience sample of mothers ( $n$ 1484) with infants aged 12 months or less, Kuwait, July-October 2015

\begin{tabular}{|c|c|c|c|c|c|}
\hline Independent variable & $B$ & SE & OR & $95 \% \mathrm{Cl}$ & Significance \\
\hline \multicolumn{6}{|l|}{ Employment prior to delivery } \\
\hline Full-time employed & 0.110 & 0.165 & $1 \cdot 12$ & $0.81,1.54$ & $P=0.506$ \\
\hline Part-time employed & -1.097 & 0.260 & 0.33 & $0.20,0.56$ & $P<0.001$ \\
\hline Student & 0.253 & 0.306 & 1.29 & $0.71,2.35$ & $P=0.409$ \\
\hline Unemployed & \multirow{2}{*}{\multicolumn{5}{|c|}{ Reference }} \\
\hline Mode of delivery & & & & & \\
\hline Vaginal & 0.429 & 0.167 & 1.54 & $1 \cdot 11,2 \cdot 13$ & $P=0.01$ \\
\hline $\begin{array}{l}\text { Elective caesarean section } \\
\text { Emergency caesarean section }\end{array}$ & $0 \cdot 106$ & \multicolumn{3}{|c|}{ Reference } & $P=0.708$ \\
\hline Information provided by nurses & & & & & \\
\hline $\begin{array}{l}\text { Yes } \\
\text { No }\end{array}$ & 0.933 & \multicolumn{3}{|c|}{ Reference } & $P=0.026$ \\
\hline Sex of the child & & & & & \\
\hline $\begin{array}{l}\text { Male } \\
\text { Female }\end{array}$ & \multicolumn{4}{|c|}{ Reference } & $P=0.026$ \\
\hline Nationality & & & & & \\
\hline $\begin{array}{l}\text { Non-Kuwaiti } \\
\text { Kuwaiti }\end{array}$ & 0.782 & 0.155 & \multicolumn{2}{|c|}{ Reference } & $P<0.001$ \\
\hline
\end{tabular}

Dependent variable: breast-feeding at discharge (1).

Cases included in the analysis: $n 1018$.

Omnibus test of model coefficients: $X^{2}=75.432$; df $8 ; P<0.001$.

Nagelkerke pseudo $r^{2}=10.4 \%$.

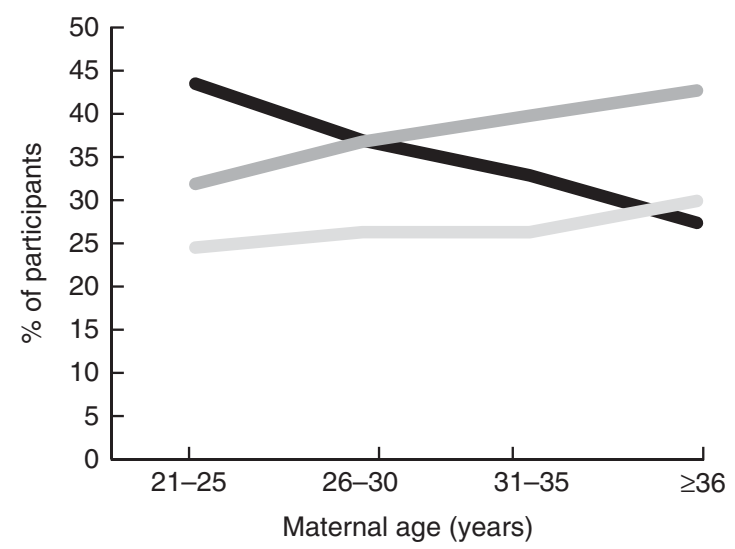

Fig. 1 Effect of maternal age on breast-feeding pattern at discharge from hospital ( $\longrightarrow$, fully breast-feeding; partially breast-feeding; , fully formula-feeding) among a convenience sample of mothers ( $n$ 1484) with infants aged 12 months or less, Kuwait, July-October 2015

had had skin-to-skin contact with their baby soon after delivery, and those who did were more likely to be fully rather than only partially breast-feeding at discharge from hospital (Table 1 and Fig. 2).

\section{Weaning}

Approximately $10 \%$ of the mothers who had stopped breast-feeding completely did so within $3 \mathrm{~d}$ postpartum, and $87.6 \%$ had stopped breast-feeding by 3 months postpartum. The mean age at complete weaning among those who had stopped breast-feeding was $49 \mathrm{~d}$ ( $<2$ months).

Decisions as to when to introduce other foods or stop breast-feeding were based on a range of real and perceived reasons. The main reasons given for stopping

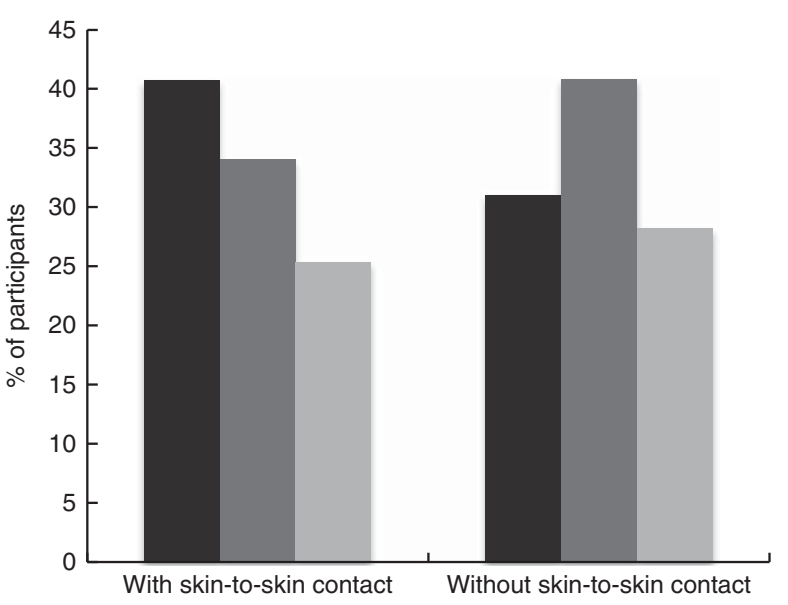

Fig. 2 Effect of skin-to-skin contact on breast-feeding pattern at discharge from hospital ( $\square$, fully breast-feeding; $\square$, partially breast-feeding; $\square$, fully formula-feeding) among a convenience sample of mothers ( $n$ 1484) with infants aged 12 months or less, Kuwait, July-October 2015

breast-feeding are summarised in Table 3 and are in keeping with reasons found in other studies, namely mothers' concern that their babies are not getting enough milk for either maternal production reasons or because they are not suckling well enough.

\section{Attitudes to breast-feeding}

In all, $70.6 \%$ of the mothers said they had found the experience of breast-feeding enjoyable and $74.1 \%$ said they would be very likely to breast-feed again. Almost all (94.2\%) the mothers who had already stopped breast-feeding said they wished they had breast-fed for longer. 
Table 3 Reasons given for ceasing breast-feeding among a convenience sample of mothers ( $n$ 1484) with infants aged 12 months or less, Kuwait, July-October 2015

\begin{tabular}{lc}
\hline Reason & $\begin{array}{c}\text { Mothers listing this } \\
\text { reason (\%) }\end{array}$ \\
\hline Baby was not getting enough milk & $50 \cdot 6$ \\
Baby was getting milk either too slowly or in & $21 \cdot 0$ \\
$\quad$ insufficient quantities & $20 \cdot 8$ \\
Baby was not sucking well & $16 \cdot 5$ \\
Formula-feeding was more convenient & $14 \cdot 5$ \\
Formula-feeding was easier & $14 \cdot 7$ \\
Breast-feeding was stressful & $13 \cdot 9$ \\
Returning to work or study soon after birth & 11.5 \\
Health or medication reasons & $8 \cdot 5$ \\
Wanted to go on oral contraceptive pill & $7 \cdot 6$ \\
Breast-feeding was painful & $6 \cdot 8$ \\
Baby was not gaining enough weight & $5 \cdot 6$ \\
Baby was too tired to feed & $3 \cdot 5$ \\
Disliked breast-feeding & $2 \cdot 6$ \\
Breast-feeding was embarrassing & $0 \cdot 8$ \\
Baby was getting either too much milk or & \\
too fast & $0 \cdot 2$ \\
Partner did not like me to breast-feed in & \\
public & $10 \cdot 2$ \\
Other (including travel, depression, baby & \\
$\quad$ refused) & \\
\hline
\end{tabular}

\section{Discussion}

The value of breast-feeding for the health of both the infant and mother is now well established ${ }^{(6)}$. In Kuwait, where the prevalence of both obesity and type 2 diabetes mellitus is growing rapidly ${ }^{(20)}$, and where age at first diagnosis of these two diseases is falling, the clinical and public health role of breast-feeding takes on even greater significance.

Breast-feeding status at the time of discharge can be an important indicator of the extent to which breast-feeding was promoted and supported in the hospital setting. The relatively low rate of breast-feeding - both exclusive and partial - seen in the present study is thus of major concern. Although most mothers said they successfully initiated breast-feeding (98.1\%), only $74 \%$ of them were breastfeeding in any form (with only $37 \%$ fully breast-feeding) by the time they left hospital. These results match those found in similar studies in Kuwait and other GCC countries ${ }^{(23,24)}$. In a previous Kuwaiti study, over $90 \%$ of mothers initiated breast-feeding but only $30 \%$ continued to fully breast-feed after discharge from hospital ${ }^{(23)}$. Data from a study in the United Arab Emirates showed that 98\% of Emirati mothers initiated breast-feeding but only $25 \%$ did so exclusively ${ }^{(27)}$. The Eastern Mediterranean Regional Office of WHO has also previously reported high rates (>60\%) of breast-feeding initiation, but at 4 months this had fallen in Lebanon (7\%), Yemen (15\%), Pakistan (16\%), Jordan (32\%) and Iran $(48 \%)^{(28)}$.

Most mothers said they enjoyed breast-feeding and most (94\%) regretted having stopped when they did. They also said they would like to breast-feed any future children. These positive attitudes are encouraging and suggest that better rates could be achieved with additional support.
The most frequent reasons mothers gave for stopping breast-feeding were belief their babies were not growing well, fear that their babies were not getting enough milk and feeling that they themselves were not producing enough milk. Studies in other parts of the world have reported similar findings ${ }^{(27,29)}$ and point to a deficiency in information and support provided by health-care staff and others. Given that previous studies in Kuwait have reported similar findings ${ }^{(24,30)}$, the value of early provision of sound information and robust counselling on infant nutrition should not be underestimated.

In the present study, paediatricians and then grandparents were named as the main sources of information mothers had on how to feed their babies. Although nurses were not very frequently mentioned as a key source of guidance, the fact remains that when they were seen as a key source of information, their guidance was very effective. Clearly much more needs to be done to provide nurses with the training and incentives that would encourage them to be more proactive in promoting and supporting breast-feeding.

Immediate postnatal skin-to-skin contact has long been promoted as a means of establishing a bond between mother and baby and also as a way of facilitating suckling by the baby ${ }^{(26,31)}$. However, less than $60 \%$ of women interviewed had had skin-to-skin contact soon after delivery and promotion of this therefore needs to be strengthened as a routine practice in all clinics.

In many post-industrial countries, breast-feeding is becoming more common among more educated mothers, suggesting that they are the most able to access and use information about the benefits of breast-feeding. In Kuwait, this was not the case. No significant association was found between breast-feeding at discharge and level of maternal education in the current study. This suggests that other external-to-the-mother factors are at work in determining breast-feeding and infant feeding behaviour. Other investigations of breast-feeding determinants in Kuwait, however, did find an association between higher education and breast-feeding for more than 6 months ${ }^{(24)}$, as did other research from the region ${ }^{(27)}$. In the present study, nationality was found to be a key determinant of breast-feeding behaviour, with Kuwaiti mothers being less likely to breast-feed. This may be indicative of different cultural and religious attitudes towards breast-feeding and young child care in general. However, it may also reflect different socio-economic circumstances and associated lifestyles of national and expatriate populations in Kuwait. Further qualitative research into the basis of this result could provide valuable insights into the cultural and religious dimensions of breast-feeding in the GCC region.

The fact that mothers with male babies were more likely to be still breast-feeding at the time of discharge suggests that mothers are aware of the value of breast-feeding and prioritise male infants. This has been observed elsewhere and calls for more attention to be given to the early care of girls. 
The need to return to work was one of the main reasons mothers gave for terminating breast-feeding in the present study. Few countries have maternity leave durations that are in keeping with the WHO recommendation of 6 months exclusive breast-feeding. Kuwait is no exception to this, and although the current study did not look specifically at eligibility for maternity leave, there was a clear tendency for mothers who had been in full-time employment in the 6 months prior to delivery to fully breast-feed. Mothers who had been part-time employed were more likely to be already introducing formula-feeding by the time of discharge. In Kuwait where most of the female expatriate labour force is engaged in the informal sector, maternity leave protection is probably not available to many.

The easy availability of breast-milk substitutes in Kuwait is a potentially adverse factor and the presence of these products in maternity wards is in direct conflict with the International Code on Marketing of Breast-milk Substitutes $^{(2)}$. This, and the fact that breast-milk substitutes are being heavily subsidised, run counter to the interests of babies and calls for attention at a governmental level.

Finally, if Kuwait is to tackle its growing diabetes epidemic it must do so comprehensively: in promoting and facilitating good infant and young child feeding practices, it will take a step in the right direction.

\section{Acknowledgements}

Acknowledgements: The researchers acknowledge the contribution of Kuwait University medical students in performing survey interviews. Financial support: Research was funded by the Dasman Diabetes Institute, based on successful application to its Scientific Advisory Board. Dasman Diabetes Institute research staff participated in the design, analysis and writing of this article. Conflict of interest: The authors declare no competing interests. Authorship: M.C. was the Principal Investigator for the project. He conceptualized the study and coordinated all aspects including its development, implementation and write-up of results. N.K. managed the project locally in Kuwait. She assisted in the development of research protocols and tools, and oversaw the collection of survey data. E.C.M. assisted in the development of research protocols, analysed results and drafted the initial paper. A.M. and S.A. assisted with the local management of the project. N.A.-H. and R.A.-W. provided guidance on development of the study and on previous breast-feeding initiatives in Kuwait. All authors contributed to the reviewing and editing of the research paper. Ethics of human subject participation: This study was conducted according to the guidelines laid down in the Declaration of Helsinki and all procedures involving human subjects/patients were approved by the Dasman Diabetes Institute Ethical Review Committee. Written informed consent was obtained from all subjects/patients.

\section{References}

1. Carballo M (1977) WHO collaborative studies on breastfeeding. J Biosoc Sci 9, 83-89.

2. World Health Organization (1981) International Code of Marketing of Breast-milk Substitutes. Geneva: WHO.

3. World Health Organization (1985) The Quantity and Quality of Breast Milk. Report on the WHO Collaborative Study on Breast-Feeding. Geneva: WHO.

4. Ip S, Chung M, Raman G et al. (2007) Breastfeeding and maternal and infant health outcomes in developed countries. Evid Rep Technol Assess (Full Rep) 153, 1-186.

5. Agarwal M, Ghousia S, Konde S et al. (2012) Breastfeeding: nature's safety net. Int J Clin Pediatr Dent 5, 49-53.

6. Victora CG, Bahl R, Barros AJD et al. (2016) Breastfeeding in the 21st century: epidemiology, mechanisms, and lifelong effects. Lancet 387, 475-490.

7. World Health Organization (2003) Global Strategy for Infant and Young Child Feeding. Geneva: WHO.

8. Rollins NC, Bhandari N, Hajeebhoy N et al. (2016) Lancet Breastfeeding Series: Why invest, and what it will take to improve breastfeeding practices in less than a generation. Lancet 387, 491-504.

9. Pettitt DJ, Forman MR, Hanson RL et al. (1997) Breastfeeding and incidence of non-insulin-dependent diabetes mellitus in Pima Indians. Lancet 9072, 166-168.

10. Owen CG, Martin RM, Whincup PH et al. (2006) Does breastfeeding influence risk of type 2 diabetes in later life? A quantitative analysis of published evidence. Am J Clin Nutr 84, 1043-1054.

11. Pereira PF, Alfenas Rde C \& Araújo RM (2014) Does breastfeeding influence the risk of developing diabetes mellitus in children? A review of current evidence. J Pediatr (Rio J) 90, 7-15.

12. Horta B \& Victora CG (2013) Long-Term Effects of Breastfeeding: A Systematic Review. Geneva: WHO.

13. Horta B, Bahl R, Martines J et al. (2013) Evidence on the Long-Term Effects of Breastfeeding. Geneva: WHO.

14. Peng H \& Hagopian W (2006) Environmental factors in the development of type 1 diabetes. Rev Endocr Metab Disord 3, 149-162.

15. Schwarz EB, Brown JS, Creasman JM et al. (2010) Lactation and maternal risk of type 2 diabetes: a population based study. Am J Med 123, 863.e1-e6.

16. Stuebe AM, Rich-Edwards JW, Willett WC et al. (2005) Duration of lactation and incidence of type 2 diabetes. JAMA 294, 2601-2610.

17. Ziegler AG, Wallner M, Kaiser I et al. (2012) Long-term protective effect of lactation on the development of type 2 diabetes in women with recent gestational diabetes mellitus. Diabetes 61, 3167-3171.

18. Oakley LL, Renfrew MJ, Kurinczuk JJ et al. (2013) Factors associated with breastfeeding in England: an analysis by primary care trust. BMJ Open 3, e002765.

19. Cai X, Wardlaw T \& Brown DW (2012) Global trends in exclusive breastfeeding. Int Breastfeed J 7, 12.

20. International Diabetes Federation (2015) IDF Diabetes Atlas, 7th ed. Brussels: IDF; available at http://www. diabetesatlas.org

21. Ziyab AH, Mohammad A, Maclean E et al. (2015) Diabetes: a fast evolving epidemic. Kuwait Med J 47, 291-301.

22. Peduzzi P, Concato J, Kemper E et al. (1996) A simulation study of the number of events per variable in logistic regression analysis. J Clin Epidemiol 49, 1373-1379.

23. Dashti M, Scott JA, Edwards CA et al. (2010) Determinants of breastfeeding initiation among mothers in Kuwait. Int Breastfeed J 5, 7.

24. Nassar MF, Abdel-Kader AM, Al-Refaee FA et al. (2014) Breastfeeding practice in Kuwait: determinant of success and reasons for failure. East Mediterr Health J 20, 409-415. 
25. Osman H, El Zein L \& Wick L (2009) Cultural beliefs that may discourage breastfeeding among Lebanese women: a qualitative analysis. Int Breastfeed J 4, 12.

26. Oras $\mathrm{P}$, Thernstrom Blomgvist $\mathrm{Y}$, Hedberg Nygvist $\mathrm{K}$ et al. (2016) Skin-to-skin contact is associated with earlier breastfeeding attainment in preterm infants. Acta Paediatr 105, 783-789.

27. Radwan H (2013) Patterns and determinants of breastfeeding and complementary feeding practices of Emirati mothers in the United Arab Emirates. BMC Public Health 25, 171.
28. Daifellah A M, Juaid AI, Colin W et al. (2014) Breastfeeding in Saudi Arabia: a review. Int Breastfeed J 9, 1.

29. Odom EC, Li R, Scanlon KS et al. (2013) Reasons for earlier than desired cessation of breastfeeding. Pediatrics 131, e726-e732.

30. Ebrahim B, Al Enezi H, Al Turki M et al. (2011) Knowledge, misconceptions, and future intentions towards breastfeeding among female university students in Kuwait. J Hum Lact 27, 358-366.

31. Biancuzzo M (2016) Breastfeeding in the past 20 years milestones to celebrate, work to be done. Nurs Womens Health 20, 135-137. 